\title{
UNIDAD DE INVESTIGACIÓN EN HOSPITALES AUTOGESTIONADOS
}

\section{RESEARCH UNIT IN SELF-MANAGED HOSPITALS}

\author{
Fredy Seguel P. ${ }^{*}$ \\ Tatiana Paravic K. ${ }^{* *}$
}

\begin{abstract}
RESUMEN
El propósito de este trabajo es reflexionar respecto de la importancia de la investigación en el centro asistencial autogestionado. Se describe el contexto sociopolítico internacional que contribuye a la formación de centros de salud autónomos en red para disminuir las desigualdades. Analiza la necesidad de incluir a la investigación como una unidad o comité dentro de las estructuras de los hospitales autogestionados para proporcionar una atención de calidad. Finalmente destaca la necesidad de que la población de enfermeras/os participe de los comités de investigación en la Unidad de Gestión del Cuidado del establecimiento con el objetivo de innovar en las prácticas, mejorar los estándares de calidad del centro asistencial y sustentar la profesión
\end{abstract}

Palabras clave: Administración hospitalaria, investigación en los servicios de salud, autonomía.

\begin{abstract}
The purpose of this paper is to reflect the importance of research in self-managed health center. It describes the international socio-political context which contributes to the formation of autonomous health centers in a network to reduce inequalities. It discusses the need to include research as a unit or committee within the structures of self-managed hospitals to provide quality care. Finally it emphasizes the need for the population of nurses involved in research committees in the Care Management Unit of the establishment with the aim of innovating practices, improve quality standards in the health center and support the profession.
\end{abstract}

Key words: Hospitals administration, research in health services, autonomy.

Fecha recepción: 09.08.10 Fecha aceptación: 06.06.11

\section{INTRODUCCIÓN}

Las relaciones entre las naciones permiten, junto con estrechar lazos y firmar acuerdos, intercambiar conocimientos, progreso y crear fuentes de trabajo de acuerdo a las demandas del mercado (1). El mercado de salud es regulado por el poder adquisitivo y los recursos disponibles para las atenciones con marcadas diferencias en lo público y privado; en este sentido no es la misma calidad en las prestaciones entre un hospital público y una clínica privada en cuanto a oportunidad del diagnóstico, tratamiento, pronóstico y rehabilitación. La desigualdad se mantiene a pesar que en muchos países los indicadores en salud han mejorado $(2,3)$. Sin embargo, los estados han tomado conciencia de esta problemática, y basados en compromisos

\footnotetext{
* Enfermero, alumno de Doctorado, Departamento de Enfermería, Facultad de Medicina, Universidad de Concepción, Chile. Email: fredyseguel@uach.cl

** Enfermera, Doctora en enfermería, Magíster en Enfermería y Salud Comunitaria, Prof. Titular Departamento de Enfermería. Universidad de Concepción, Chile.Email: tparavic@udec.cl
} 
internacionales se esfuerzan por estrechar la brecha, creando la figura de hospitales públicos autónomos o autogestionados aislados o conformando una red de establecimientos que pretenden sostener cabalmente las necesidades en salud de los individuos (3-5). Son características fundamentales de estas instituciones de salud la descentralización de poderes desde los Servicios de Salud a las Direcciones Hospitalarias, la acreditación de éstos de acuerdo a estándares estrictos de calidad y seguridad del paciente, la adopción de procesos de gestión claros con monitoreo constante, rendición de cuentas e innovación continua $(4,6)$. La Real Academia Española define autogestión como el "sistema de organización de una empresa según el cual los trabajadores participan en todas las decisiones", el proceso democrático está implícito donde la información debe fluir vertical y horizontalmente. El capital humano es el motor de la autogestión, donde la calidad integral del ambiente de trabajo hace la diferencia mejorando la productividad (7). Esto exige reformas políticas y sociales con instauración sólida de la investigación para confirmar o refutar las prácticas a través de las cuales se logra una atención adecuada (8).

El propósito del presente trabajo es fundamentar la importancia de la investigación en los hospitales autogestionados como pilar esencial en la gestión y aseguramiento de la calidad de las prestaciones, mejorando la eficiencia y eficacia de las acciones así como la seguridad y la satisfacción del usuario.

\section{ESCENARIO SOCIOPOLÍTICO EN LA CREACIÓN DE HOSPITALES AUTOGESTIONADOS}

En el siglo XX se afianzó el concepto de globalización con las reformas en el ámbito económico manteniendo las desigualdades entre los niveles sociales. Los estados disminuyeron su protagonismo y se han produ- cido grandes crisis económicas a manos de empresas multinacionales. El desarrollo de la dispersión global en la producción ha significado el surgimiento de la flexibilidad en los empleos, caracterizándose por la aparición de trabajos a honorarios, independientes y temporales (9), con la consecuente disminución del valor de la mano de obra, aumento de la competencia, riesgos en los puestos de trabajo y las enfermedades en los trabajadores (10). Además, las necesidades de las familias crean un nicho para que las mujeres ingresen progresivamente al mundo del trabajo, ejemplo de ello es la agroindustria donde existe un gran porcentaje de trabajadoras temporales (11). Las migraciones, pobreza, urbanización, contaminación de nuestro entorno, relaciones internacionales y cambios en nuestros estilos de vida son factores importantes en el comportamiento de este modelo que han acarreado consecuencias como la proliferación de ciertas enfermedades tales como síndrome de inmuno deficiencia adquirida, tuberculosis, infecciones respiratorias y enfermedades tropicales; ébola, SARS, dengue entre otros $(11,12)$.

Los índices del banco mundial muestran las brechas que existen en los países, la participación en el ingreso en el $20 \%$ de la población peor remunerada es de 7,34\% versus el $20 \%$ mejor remunerada con un $43,7 \%$ (13). La expectativa de vida bordea los 44 años en el país más pobre, la tasa de mortalidad infantil es de 165 por mil nacidos vivos respecto de 1,2 por mil nacidos vivos en el más enriquecido y la tasa de mortalidad en menores de 5 años es de 257 por cada mil nacidos vivos respecto de 1,9 por cada mil nacidos vivos de los países más ricos (14). Esto llevó a que los estados examinaran alternativas de mejora en la equidad de servicios, descentralizando su poder político y de responsabilidad hacia los centros hospitalarios, buscando su autonomía en la gestión y eficiencia en la distribución de recursos públicos, sin embargo en muchas ocasiones no se obtuvieron los resultados deseados $(4,15,16)$. 
Un factor importante a considerar en la mejoría de ciertos índices en salud es la investigación, que contribuiría a elevar la calidad de vida y el nivel de educación para la formación de personas y paralelamente ayudaría a entender y relacionar integralmente los últimos adelantos en el conocimiento, dotando a los agentes gubernamentales de mayor capacidad de diálogo, colaboración y negociación. Una decisión importante tendrá menos posibilidad de error y será más eficiente y eficaz si se han analizado los estudios que existen al respecto y los factores que intervienen en su aplicabilidad (17). Por otro lado, la diseminación del conocimiento y el aumento sustancial en el desarrollo tecnológico permiten innovar en todo ámbito, en el sector salud se ha avanzado a pasos agigantados pues la investigación científica ha logrado poner a disposición de los tratamientos y del cuidado la más alta tecnología para solucionar enfermedades complejas (18).

\section{REFORMA DE SALUD, GESTIÓN E INVESTIGACIÓN EN LOS HOSPITALES AUTOGESTIONADOS EN CHILE}

Chile mantiene marcada influencia de la globalización. La constante evolución del avance científico y económico ha permitido mayor inversión en el aspecto social y trabajo, aumentando la expectativa de vida de los ciudadanos, pero manteniendo las inequidades. A pesar de ello, los estándares en salud han mejorado sustancialmente en comparación a 20 años atrás (19). El estado, a juicio de los autores de este trabajo, ha realizado cambios radicales en el sector en pos de estrechar las brechas de inequidad y mejorar la atención especialmente en las áreas más pobres, pero no se evidencian políticas de investigación formalmente establecidas que faculten a los centros asistenciales autogestionados a la formación de comités o unidades de investigación para innovar y fundamentar con evidencias, así como intercambiar éstas con otros establecimientos nacionales e internacionales.

En el contexto de las desigualdades y de las políticas de mejora en el sector, surge el proceso de acreditación hacia la autogestión de los hospitales, requisito obligatorio para obtener recursos financieros, respaldado por la implementación de las garantías explícitas en salud que en estos momentos comprenden 66 patologías, garantizando el diagnóstico, tratamiento y rehabilitación, basados en evidencia científica fundada (20). Estos centros asistenciales son definidos como aquellos de alta complejidad técnica que cuentan al menos con alguna especialidad básica (medicina interna, obstetricia-ginecología y pediatría) y con diversas subespecialidades (ej.: cardiología, neurología, nefrología, gastroenterología, oftalmología, otorrinolaringología). Las características del modelo están basadas en la descentralización de la gestión y su funcionamiento mediante altos estándares de calidad (21).

Los requisitos mínimos que deben cumplir las instituciones de salud para la obtención de la calidad de autogestionados están orientados a evidenciar su adecuada participación y articulación en la red asistencial, registro vigente en la superintendencia de salud como prestador de servicios acreditado, tener un plan de desarrollo integral en concordancia con las políticas y normativas de la red asistencial del servicio. Este debe hacer referencia a los recursos humanos, gestión financiera, clínica, administrativa, estrategias de recaudación, auditoría interna, sistemas de planificación, mantener un plan anual de inversiones y de actividades basados en el plan estratégico, salvaguardar el equilibrio financiero entre ingresos y egresos, mantener métodos de evaluación de costos, mantener cumplimiento de las Garantías Explícitas en Salud, tener implementados los métodos de cobro y recaudación de ingresos, explicitar mecanismos formales de participación, mantener dispositivos serios especializados 
que atiendan las sugerencias, reclamos y felicitaciones con el objetivo de dar solución (21).

Se definen también las funciones que deben desempeñar estos establecimientos de acuerdo a su denominación dando respuestas amplias a la red asistencial en cuanto a los beneficiaros, atención cerrada y ambulatoria, los sistemas de información, sistemas de registro, convenios, auditorías y el financiamiento que comprende, entre otros, la presentación de proyectos a fondos concursables y otros aportes (21), por lo tanto, la investigación puede además contribuir monetariamente al desarrollo del centro.

En la acreditación para la autogestión (22), se toca explícitamente la dignidad del paciente, gestión de la calidad propiamente tal, gestión clínica, acceso, oportunidad y continuidad de la atención, competencias del recurso humano, registros, seguridad del equipamiento y seguridad de las instalaciones. En este sentido el establecimiento debe contar con una política bien documentada, con registros definidos tanto para su promoción como su evaluación, indicadores elaborados de acuerdo a las falencias encontradas y una persona responsable de las actividades que se deben poner en marcha (23).

Entre los problemas que se han previsto en la implementación de este sistema en Chile, los más importantes son: la percepción de la insatisfacción usuaria, los sistemas de información complejos y la inexistencia formal de unidades de investigación para la fundamentación e innovación de las actividades clínicas. En esta última, la recomendación es realizar investigación combinada entre metodología cualitativa y cuantitativa para evidenciar detalladamente los procesos que se están efectuando (24).

La investigación en el centro asistencial proporciona un aumento del funcionamiento en general, eficiencia a nivel local, regional y nacional; asegura la calidad de atención, el rendimiento clínico y la racionalización de los procesos de decisión $(25,26)$. Existen ejemplos en el desarrollo del centro hospitalario en torno a la investigación generando recursos frescos para la gestión; tal es el caso del hospital clínico de Barcelona que en un periodo de 40 años, reformulando su estructura funcional y partiendo con deficiencias en la producción científica, logró llegar al primer lugar en publicaciones de todos los hospitales de España en el año 2002. El cambio fue paulatino y dentro de sus objetivos de gestión estaba la investigación, se creó un centro de investigación de ciencias básicas dependiente del estado local en Cataluña, del centro asistencial y de la Universidad de Barcelona, con personas de vasta experiencia en apoyo de la práctica clínica. Con esto lograron sobrepasar los 500 artículos originales en el año 2005, con un factor de impacto de 2,242 puntos, ingresando alrededor de 33 millones de euros, un $60 \%$ provenía del sector público y el resto del sector privado (27). En España se han formado fundaciones de investigación las cuales han proporcionado gran apoyo para los centros hospitalarios, sin embargo, se reconoce que la investigación en general, no necesariamente clínica, históricamente ha sido la actividad menos realizada y que se han tenido que efectuar grandes esfuerzos para lograr aumentar la evidencia que mejore la práctica (28).

En este modelo de autogestión hay una población funcionaria importante que puede aportar en investigación mejorando los procesos de atención de cuidados en los centros hospitalarios y éstos son los profesionales de enfermería situados en la organización de la Unidad de Gestión del Cuidado de dependencia directa del director del establecimiento y cuyas funciones son transversales en los diferentes servicios, y cuyo objetivo principal es mejorar la calidad y seguridad de la atención del usuario así como también debe innovar en las prácticas asistenciales basadas siempre en las evidencias científicas, por lo que necesita de la investigación para beneficio directo de los pacientes. La capacidad de identificar las falencias de conoci- 
miento en áreas prioritarias se transforma en herramienta precursora de la necesidad de buscar conocimiento o generarlo a partir de una pregunta de investigación, siempre y cuando sea realizado con rigurosidad (29). Las enfermeras están obteniendo experiencia en la investigación cuantitativa y cualitativa a través de sus estudios curriculares de pre y postgrado. En este sentido, en América han surgido focos geográficos de generación de conocimiento con múltiples publicaciones y creación de revistas especializadas en los saberes de la disciplina, lo que potencia el posicionamiento de la profesión, sin embargo, una de las críticas que surgen es que la investigación no está nutriendo a la profesión de enfermería.

Por un lado, aún las publicaciones en enfermería son escasas (29), y por otro, muchas de las investigaciones que se han publicado no han tenido la fuerza suficiente para implementar dichos hallazgos en el nivel normativo ni en el trabajo diario de su profesión, a pesar de que es parte del compromiso social que la enfermera o enfermero debe tener con la sociedad para mejorar la calidad de atención (30). Pero en la medida que exista un comité dentro de las Unidades de Gestión del Cuidado, podrán participar en la definición de políticas y lineamientos estratégicos de la institución que permitan un mejoramiento continuo del cuidado, dando énfasis a la solución de problemas asistenciales mediante la investigación e implementando estos resultados en la calidad de atención hacia el usuario, y en el sustento hacia la profesión.

\section{CONSIDERACIONES FINALES}

Es claro que la investigación es importante para mejorar la calidad de las prestaciones en salud y que la formación de hospitales autónomos responde a la necesidad de optimización de la gestión en el contexto de la globalización para lograr la igualdad de oportunidades de los quintiles más pobres en comparación con el sector privado.

La investigación no se ha posicionado a cabalidad en muchos de los centros asistenciales y no ha ido de la mano con el desarrollo de la autogestión, en estos momentos se están aplicando las normativas emitidas por el gobierno central y evaluando lo que ha estado realizando por muchos años, con el objetivo de determinar el cumplimiento de ciertos estándares, lo que debiera ser un proceso de transición hasta obtener indicadores óptimos.

No se expresa en la legislación la formación de comités de investigación o redes que involucren a universidades acompañando el proceso, como lo sucedido con otros países; lo que potenciaría el diseño de programas adecuados para enfrentar los desafíos de la autogestión.

En este sentido los profesionales de enfermería bajo la Unidad de Gestión del Cuidado pueden tener protagonismo en investigación, relacionado con la gestión de los servicios y los cuidados para con los pacientes, ya que su campo de acción los sitúa prácticamente en todas las áreas de especialidades y subespecialidades.

Enfermería debe buscar estrategias que le permitan tener la fuerza suficiente para implementar en la práctica los diversos hallazgos provenientes de las múltiples investigaciones realizadas que constituyen evidencias de calidad e innovación, permitiendo funcionar a los hospitales autogestionados en los más altos estándares de calidad.

\section{REFERENCIAS}

1. De Cárdenas A, Jiménez N. Acceso universal a la información: globalización, cultura y alfabetización. ACIMED. 2007; 15(1).

2. Alonso P. Tendencias de la salud internacional: retos y oportunidades para nuestra sociedad. Gac Sanit. 2003;17(3):175-8. 
3. Guzmán M. Deficiencias en los diagnósticos de las reformas sanitarias de los años noventa en América Latina. Rev Panam Salud Pública. 2009;25(1):84-92.

4. Méndez CA, Torres AMC. Hospital management autonomy in Chile: the challenges forhuman resources in health. Rev Saude Publica. 2010; 44(2): 366-71.

5. Bossert T, Beauvais J. Decentralization of health sistem in Ghana, Zambia, Uganda and the Philippines: a comparative analysis of decision space. Health Policy Plan. 2002;17(1):1431.

6. Bowman CE. Governance and autonomy in alternatives to hospital care. Age Ageing. 2001;30 (3supl):15-8.

7. Alarcón A. El tránsito a la autogestión hospitalaria en Chile. Revista Chilena de Salud Pública. 2009;13(3):125-7.

8. Cardona A, Nieto E, Restrepo R, Sierra O, Cárdenas CE, Aguirre F. Una propuesta de innovación en políticas de aseguramiento en salud, basada en hallazgos de investigación: el caso de los trabajadores cesantes en Medellín, Colombia. Cad Saude Publica. 2006;22(supl):87-96.

9. Kawachi I. Globalization and workers' health. Ind Health. 2008 Oct;46(5):4213.

10. Kogi K, Hisanaga N, Araki S. Good practices in occupational safety and health in the new era of globalization. Ind Health. 2008 Oct;46(5):419-20.

11. Mora C. Globalización, género y migraciones. Polis. 2008; (20):285-97.

12. Marchiori B. Globalização, pobreza e saúde. Ciênc Saúde Coletiva. 2007;12(6):1575-89.

13. Banco Mundial. [Internet]. Indicadores. [citado 30 mayo 2010]. Disponible en: http://datos.bancomundial.org/indicador. Acceso el 30 de mayo de 2010.

14. Banco Mundial. [Internet]. Tasa de mortalidad, menores de 5 años [citado 30 mayo 2010]. Disponible en: http://datos. bancomundial.org/indicador.

15. Belmartino S. Una Década de Reforma de la Atención Médica en Argentina. Salud Colec. 2005;1(2):155-71.

16. Carrioni CA, Hernández ML, Molina M. La autonomía de las instituciones prestadoras de servicios de salud (IPS): más un ideal que una vivencia institucional. Revista Facultad Nacional de Salud Pública. 2007;25(2):75-84.

17. García A. Investigación básica y poderes públicos. Rev Iberoam Cienc Tecnol y Soc. 2007;3(8):115-26.

18. Franco A. Globalizar la salud. Gac Sanit. 2003;17(2):157-63.

19. Sociedad Chilena de Administradores en Atención Médica y Hospitalaria. Los desafíos pendientes de la autogestión hospitalaria en red. Cuad Med Soc. 2009;49(3):171-7.

20. Ley 19.937 de 30 enero, 2004. Autoridad Sanitaria. Modifica el D.L. No 2.763, de 1979 , con la finalidad de establecer una nueva concepción de la autoridad sanitaria, distintas modalidades de gestión y fortalecer la participación ciudadana. Diario Oficial Chile 24 febrero 2004.

21. Decreto 38 de 2005, diciembre 29. Reglamento Orgánico de los Establecimientos de salud de menor complejidad y de los establecimientos de autogestión en red. Diario Oficial Chile 29 diciembre 2005.

22. Decreto 15 de 2007, julio 3. Reglamento del sistema de acreditación para los prestadores institucionales de salud. Diario Oficial Chile 3 de julio 2007.

23. Superintendencia de Salud. Intendencia de Prestadores en Salud. Decreto Exento 18 de 2009, marzo 19. Manual del estandar general de acreditación para prestadores institucionales de atención cerrada. Santiago, Chile; 2009.

24. Sánchez S. Hospitales autogestionados en la red de salud de Chile: alcances y limitaciones de su ley. Cuad Méd Soc. 2010;50(1):5-10.

25. Vera A, Salge TO. The impact of research and development on hospital performance - an empirical analysis in the eng- 
lish hospital sector. Gesundheitswesen. 2011; 73(3):142-8.

26. Zamberlan A. Health services research and development: a tool for hospital management. Health Serv Res. 1990;25(1 Pt 2):169-75.

27. Rodés J. La experiencia del hospital clínico de Barcelona: integración Facultad de Medicina - IDIBAPS - Hospital Universitario. Educ méd. 2007;10(4):202-8.

28. Bonilla F. Fundaciones para la investiga- ción biomédica hospitalaria [editorial]. Oncología (Barc ). 2006;29(4):11-2.

29. Harrison L, Ray A, Cianelli R, Rivera MS, Urrutia M. Competencias en investigación para diferentes niveles de formación de enfermeras: una perspectiva latinoamericana. Cienc enferm. 2005;11(1):5971.

30. Martini JG. O papel social da pesquisa em enfermagem. Rev Bras Enferm. 2009;62(3):340-42. 